\title{
Guideline for advanced neuroimaging in pediatric epilepsy
}

Yun Jung Hur, MD, PhD

Department of Pediatrics, Inje University Haeundae Paik Hospital, Inje University College of Medicine, Pusan, Korea

Constant advances in neuroimaging technology have enabled the detection of abnormal brain structures that can cause epilepsy or neurodevelopmental impairment and have provided opportunities for epilepsy surgery by providing high-quality information about the brains of patients with pediatric epilepsy. ${ }^{1,2)}$ Advanced neuroimaging using multimodal procedures is an important diagnostic tool for identifying biomarkers of epileptogenesis and drug-resistant epilepsy. Magnetic resonance imaging (MRI) identifies structural and functional changes in abnormal neuropathologic areas with postprocessing and advanced techniques. Positron emission tomography (PET) and single-photon emission computerized tomography (SPECT) provide the information for cerebral functional changes of epileptogenic areas via brain metabolism and blood perfusion. ${ }^{3)}$ Multimodal techniques such as PET-MRI, subtraction of interictal from ictal SPECT coregistered to MRI (SISCOM), electroencephalography-functional MRI (EEG-fMRI), and magnetoencephalography (MEG) can also help elucidate the functional alterations of epileptogenic areas in epilepsy. ${ }^{4)}$

When the first unprovoked seizure occurs or newly diagnosed epilepsy is confirmed, MRI should immediately be performed because structural lesion strongly indicates drug resistance in epilepsy. The International League Against Epilepsy recently provided a new consensus recommendation for the use of MRI in epilepsy. The Neuroimaging Task Force recommendation sug. gests the use of Harmonized Neuroimaging of Epilepsy Strucural Sequences (HARNESS-MRI) to better identify the structural lesions that may be in the epileptogenic region. HARNESS-MRI reveals high-resolution 3-dimensional (3D) T1-weighted imaging with isotropic millimetric voxel resolution $(1 \mathrm{~mm} \times 1 \mathrm{~mm} \times 1$ $\mathrm{mm}$ ) and no interslice gap, high-resolution 3D fluid attenuation inversion recovery (FLAIR) with isotropic millimetric voxel resolution $(1 \mathrm{~mm} \times 1 \mathrm{~mm} \times 1 \mathrm{~mm})$ and no interslice gap, and high in-plane resolution 2-dimensional coronal T2-weighted imaging with submillimetric voxel resolution $(0.4 \mathrm{~mm} \times 0.4 \mathrm{~mm} \times 2$ $\mathrm{mm}$ perpendicular to the long axis of the hippocampus) and no interslice gap. ${ }^{1)}$ In patients younger than 2 years of age, it is important to consider that MRI sequences should differ due to poor myelination that appears at this time. ${ }^{2)}$ The early detection on MRI is important in pediatric epilepsy, and repeated MRI should be recommended due to ongoing myelination, particularly when patients have no structural lesions. ${ }^{5)}$ MRI using the HARNESS-MRI protocol should also be repeated when an unexplained increase in seizure frequency, rapid deterioration in cognition and development, or the appearance/exacerbation of neuropsychiatric symptoms occurs. ${ }^{1)}$ To better detect epileptogenic areas, MRI postprocessing can be applied, with which several studies reported higher positive detection rates of epileptogenic areas. ${ }^{67}$ However, it is essential to have standards with reproducibility and clinical validation.

In children with drug-resistant focal epilepsy, epilepsy surgery is an acceptable treatment; for this purpose, epileptogenic areas should be detected as precisely as possible. For epilepsy surgery, interictal/ictal EEG, video EEG, MRI, fluorodeoxyglucose (FDG) -PET, SPECT, electrocorticography, and intracranial monitoring are among the mandatory diagnostic tests used to localize epileptogenic areas. ${ }^{4)}$ FDG-PET reveals a wider hypometabolism than the epileptogenic areas, possibly indicative of functional abnormalities. Ictal/interictal SPECT reveals focal hyperperfusion/hypoperfusion of epileptogenic areas and is more suitable than PET in that aspect. Advanced neuroimaging techniques with the potential ability to detect epileptogenic areas include PET-MRI, SISCOM, EEG-fMRI, EEG source imaging, MEG, MR spectroscopy, MRI postprocessing techniques, and diffusionweighted imaging with tractography.5) EEG source imaging/ MEG helps localize epileptogenic areas as well as those with altered functional connectivity. EEG records tangential and radial currents with temporal resolution but has limited spatial resolution. EEG source imaging overcomes the limitations of EEG recordings in terms of temporal and spatial resolution. MEG records magnetic fields of tangential currents, which can be used for MRI-negative patients with suspected tangential areas in which the EEG tracing is likely to be distorted. In terms of cost effectiveness, EEG source imaging may be superior to MEG, but the EEG source imaging and MEG are complementary. MEG and fMRI can effectively detect eloquent areas in children and young children, in whom clinical assessments may be difficult and very few validation studies are available. ${ }^{8)}$

Advanced neuroimaging is a challenging tool in the field of pediatric epilepsy. It is now possible to identify the epileptogenic

Corresponding author: Yun Jung Hur, MD, PhD. Department of Pediatrics, Inje University Haeundae Paik Hospital, Inje University College of Medicine, 875 Haeun-daero, Haeundae-gu, Busan 48108, Korea 
areas in nonlesional cases and increase surgical candidates. Advanced neuroimaging can be a prognostic factor for better surgical outcomes that can replace invasive tests and provide information about the eloquent areas, reducing functional deficits that may arise due to excision of the eloquent areas. Advanced neuroimaging will be an important diagnostic tool for identifying biomarkers of epileptogenesis, altered functional connectivity, and localization of epileptogenic areas.

\section{Conflicts of interest}

No potential conflict of interest relevant to this article was reported.

See the article "Advanced neuroimaging techniques for evaluating pediatric epilepsy" in Volume 63 on page 88.

\section{References}

1. Bernasconi A, Cendes F, Theodore WH, Gill RS, Koepp MJ, Hogan RE, et al. Recommendations for the use of structural magnetic resonance imaging in the care of patients with epilepsy: a consensus report from the International League Against Epilepsy Neuroimaging Task Force. Epilepsia 2019;60:1054-68.

2. Gaillard WD, Chiron C, Cross JH, Harvey AS, Kuzniecky R, HertzPannier $\mathrm{L}$, et al. Guidelines for imaging infants and children with recentonset epilepsy. Epilepsia 2009;50:2147-53.

3. Reddy SD, Younus I, Sridhar V, Reddy DS. Neuroimaging biomarkers of experimental epileptogenesis and refractory epilepsy. Int J Mol Sci 2019;20(1). pii: E220. https://doi.org/10.3390/ijms20010220.

4. Jayakar P, Gaillard WD, Tripathi M, Libenson MH, Mathern GW, Cross $\mathrm{JH}$, et al. Diagnostic test utilization in evaluation for resective epilepsy surgery in children. Epilepsia 2014;55:507-18.

5. Lee YJ. Advanced neuroimaging techniques for evaluating pediatric epilepsy. Clin Exp Pediatr 2020;63:88-95.

6. Hong SJ, Kim H, Schrader D, Bernasconi N, Bernhardt BC, Bernasconi A. Automated detection of cortical dysplasia type II in MRI-negative epilepsy. Neurology 2014;83:48-55.

7. Wang ZI, Suwanpakdee P, Jones SE, Jaisani Z, Moosa AN, Najm IM, et al. Re-review of MRI with post-processing in nonlesional patients in whom epilepsy surgery has failed. J Neurol 2016;263:1736-45.

8. Collinge S, Prendergast G, Mayers ST, Marshall D, Siddell P, Neilly E, et al. Pre-surgical mapping of eloquent cortex for paediatric epilepsy surgery candidates: Evidence from a review of advanced functional neuroimag. ing. Seizure 2017;52:136-46. 